\title{
Dor aguda no joelho do paciente idoso
}

\author{
Acute knee pain in elderly patients
}

\author{
Gilberto Luís CAMANHO ${ }^{1}$
}

\section{RESUMO}

A dor aguda no joelho de pacientes idosos é freqüente, sendo caracterizada por seu aparecimento súbito, sem causa aparente, com exame radiográfico dentro dos padrões da normalidade, na maioria dos casos. A etiologia da dor aguda no joelho é decorrente de insuficiência das estruturas, e seu quadro clínico difere completamente daquele determinado pela osteoartrose, sendo sempre unilateral e ocorre na grande maioria em pacientes do sexo feminino, após a quinta década de vida, e na região medial do joelho (local de maior carga). Com o propósito de analisar as possíveis etiologias para a dor aguda do joelho de pacientes acima de 60 anos de idade, suas características e tratamento, os autores discutem as etiologias relacionadas à dor aguda: lesão meniscal, fratura por fadiga e osteonecrose idiopática.

Descritores - Dor/etiologia; Articulação do joelho/patologia; Osteonecrose; Meniscos tibiais/lesões; Fraturas de estresse; Idoso

\section{ABSTRACT}

Acute knee pain in elderly patients is not uncommon, and is characterized by a sudden onset, no apparent cause, and by normal radiographic

* Trabalho realizado no Instituto de Ortopedia e Traumatologia da Faculdade de Medicina da Universidade de São Paulo - USP São Paulo (SP), Brasil.

1. Livre-Docente, Professor do Instituto de Ortopedia e Traumatologia da Faculdade de Medicina da Universidade de São Paulo IOT/FMUSP.

Endereço para correspondência: Gilberto Luís Camanho, Rua Oliveira Dias, 61 - São Paulo (SP), Brasil. Tel.: (11) 3887-9776.

E-mail: gilbertocamanho@uol.com.br

Copyright RBO2008 findings in most cases. The etiology of acute knee pain is the result of insufficient structures, and clinical symptoms are totally different from the symptoms seen in osteoarthrosis. This acute pain in the knee is always unilateral and in the medial region of the knee joint (site with the heaviest load), predominantly in females after the fifth decade of live. In order to consider the possible etiologies for acute knee joint pain in patients older than 60 years, its characteristics and treatment, the authors discuss etiologies related to acute pain: lesion of the meniscus, stress fracture, and idiopathic osteonecrosis.

Keywords - Pain/etiology; Knee joint/pathology; Osteonecrosis; Menisci, tibial/injuries; Fractures, stress; Aged

\section{INTRODUÇÃO}

A queixa de dor no joelho, em pacientes de idade superior a 60 anos, é freqüente e, em geral, é provocada pelo desequilíbrio muscular decorrente do envelhecimento ou mesmo do processo degenerativo tão comum nesses pacientes ${ }^{(1)}$. O desequilíbrio muscular caracteriza-se pela atrofia do quadríceps associada à retração dos músculos isquiotibiais, que de maneira progressiva leva ao posicionamento em flexo da articulação do joelho.

$\mathrm{O}$ processo degenerativo articular ocorre nessa faixa etária junto de manifestações clínicas freqüentes no joelho. A dor decorrente desse processo é de aparecimento progressivo e evolutivo, que piora com a atividade física e que às vezes associa-se a deformidades da articulação do joelho.

Há um grupo de pacientes, nessa faixa etária, que embora não apresente processo degenerativo impor- 
tante, relata dor no joelho de início súbito, sem causa traumática. Estes pacientes têm características próprias sob o ponto de vista clínico, etiológico e terapêutico.

O objetivo deste artigo é analisar as etiologias possíveis e o tratamento da dor aguda no joelho do paciente com faixa etária acima das sexta década de vida.

\section{QUADRO CLÍNICO}

O quadro clínico do paciente que nos procura relatando queixa de dor aguda do joelho é bem característico: início súbito, sem causa traumática; sendo que, em alguns casos, o paciente relata traumas de pouca importância relacionados ao joelho acometido.

Há certa incapacidade funcional provocada pela dor, acentuada ao caminhar e imediatamente após o repouso. A dor noturna é freqüente e alguns pacientes relatam utilizar uma almofada entre os joelhos para dormir.

Os antecedentes da dor são raros e o sintoma agudo provoca surpresa nos pacientes. Os falseios ao caminhar ocorrem com pouca freqüência; o derrame articular é excepcional e é mais comum nos pacientes do sexo feminino.

O exame físico demonstra o joelho em atitude de flexão, não muito acentuada $\left(10^{\circ}\right.$ a $\left.15^{\circ}\right)$, que ao tentarmos corrigir causa dor. A dor também é identificada durante a palpação da interlinha medial e, com menor freqüência, na lateral. A sinovite com derrame articular nem sempre pode estar presente.

A flexão do joelho acometido com o paciente em apoio monopodálico desperta dor, desconforto e insegurança.

\section{QUADRO RADIOLÓGICO}

$\mathrm{O}$ estudo por imagem possibilita o esclarecimento diagnóstico da maioria dos casos. A radiografia simples do joelho freqüentemente é normal, podendo em raros casos ser visível um traço subcondral que sugere fratura por fadiga.

A ressonância magnética (RM) é fundamental e possibilita o diagnóstico etiológico de três lesões que determinam a dor aguda no joelho: lesão meniscal (LM); osteonecrose idiopática do joelho (OIJ); fratura por fadiga $(\mathrm{FF})$.

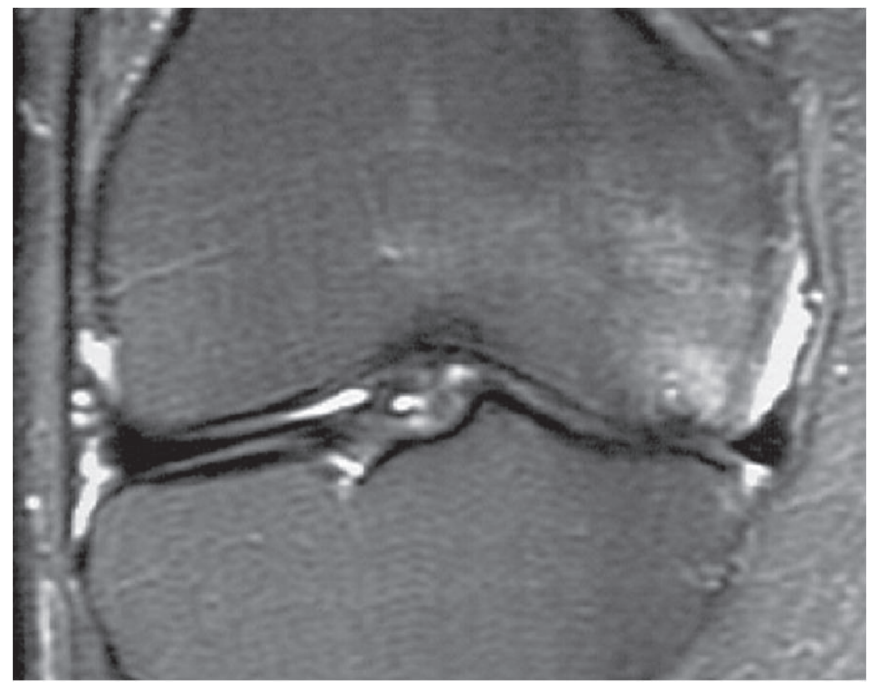

Figura 1 - RM demonstrando lesão meniscal, com o menisco subluxado e com edema ósseo no côndilo femoral

Há um grupo destes pacientes que não apresenta nenhuma alteração no exame diagnóstico por imagem.

\section{LESÃO MENISCAL}

A lesão meniscal é uma das patologias mais freqüentes do joelho, podendo ser classificada quanto à etiologia em três tipos: traumática - decorrente de um episódio traumático específico e descrito pelo paciente; degenerativa - decorrente da artrose do joelho; por fadiga - lesões de aparecimento súbito sem causa traumática evidente.

A lesão meniscal por fadiga tem os sintomas que caracterizam os pacientes do presente estudo e ocorre com maior freqüência no menisco medial de pacientes do sexo feminino ${ }^{(2)}$. O exame radiográfico simples, em geral, é normal. A RM demonstra o menisco medial subluxado na maioria dos casos. Esta luxação é provocada pela abertura do "rim" meniscal devido à presença de uma fenda radial entre a transição do corpo e o corno posterior (figuras 1 e 2). Esse tipo de lesão ocorre, ao nosso entendimento, por fadiga.

Osteonecrose idiopática do joelho é condição patológica descrita por Ahlbäck et al, que apresenta quadro clínico como o descrito pelos autores e ocorre com maior freqüência em mulheres após a sexta década de $\operatorname{vida}^{(3)}$. O comprometimento do côndilo femoral medial é muito mais freqüiente ${ }^{(4-5)}$. $\mathrm{O}$ exame radiográfico 


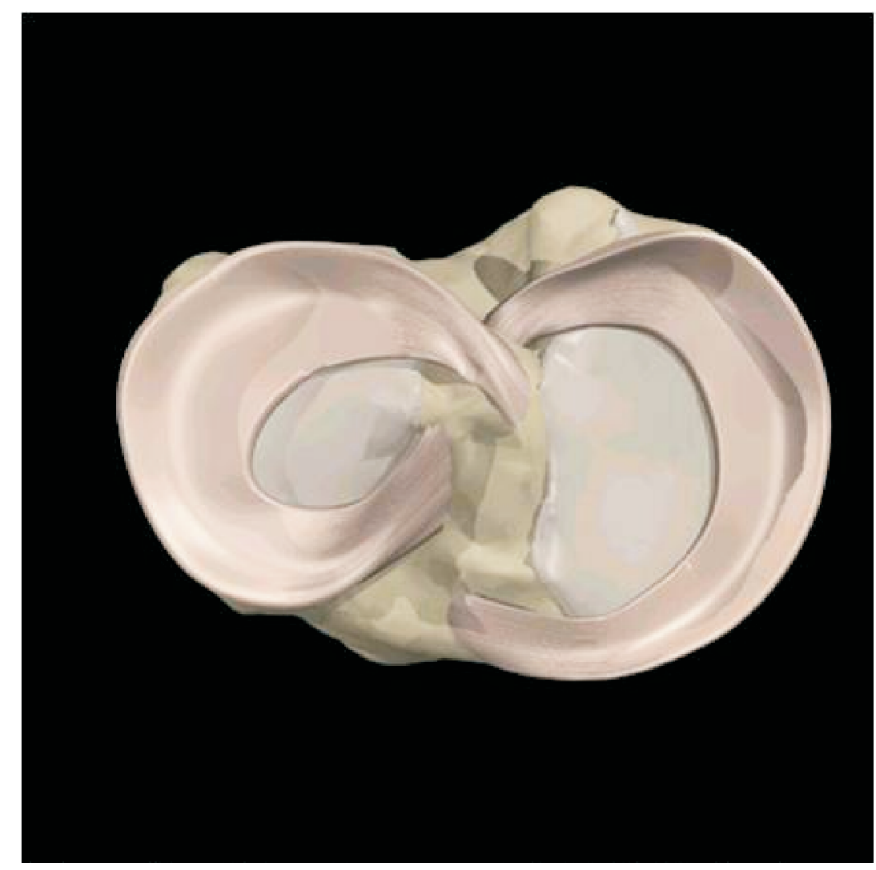

Figura 2 - Esquema demonstrando lesão meniscal na transição entre o corpo e o corno posterior

demonstra a presença de lesão, apenas nos casos mais avançados (figura 3). A RM demonstra edema ósseo e em alguns casos há sinais de traço de fratura (figura 4), que é favorável à etiologia traumática da $\mathrm{OIJ}^{(6)}$.

A fratura por estresse ou por fadiga do planalto tibial ou do côndilo femoral tem a mesma sintomatologia descrita neste artigo para as outras duas afecções. O exame radiográfico pode apresentar imagem do traço de fratura (figura 5), porém a RM é conclusiva, demonstrando o traço da fratura e o edema.

Há um pequeno grupo de pacientes com os mesmos sintomas, nos quais não há nenhuma imagem na RM que sugira lesão. Em alguns casos, há sinais de osteoporose localizada no côndilo femoral ou tibial, dependendo da resolução do equipamento de RM.

\section{TRATAMENTO}

Estas lesões são todas decorrentes de insuficiência das estruturas envolvidas. Ocorrem em geral em pacientes que apresentam osteoporose ${ }^{(7)}$.

O tratamento inicial é a proteção do joelho pelo repouso e o uso de muletas com o intuito de reduzir parcialmente a carga sobre o membro, durante a marcha.
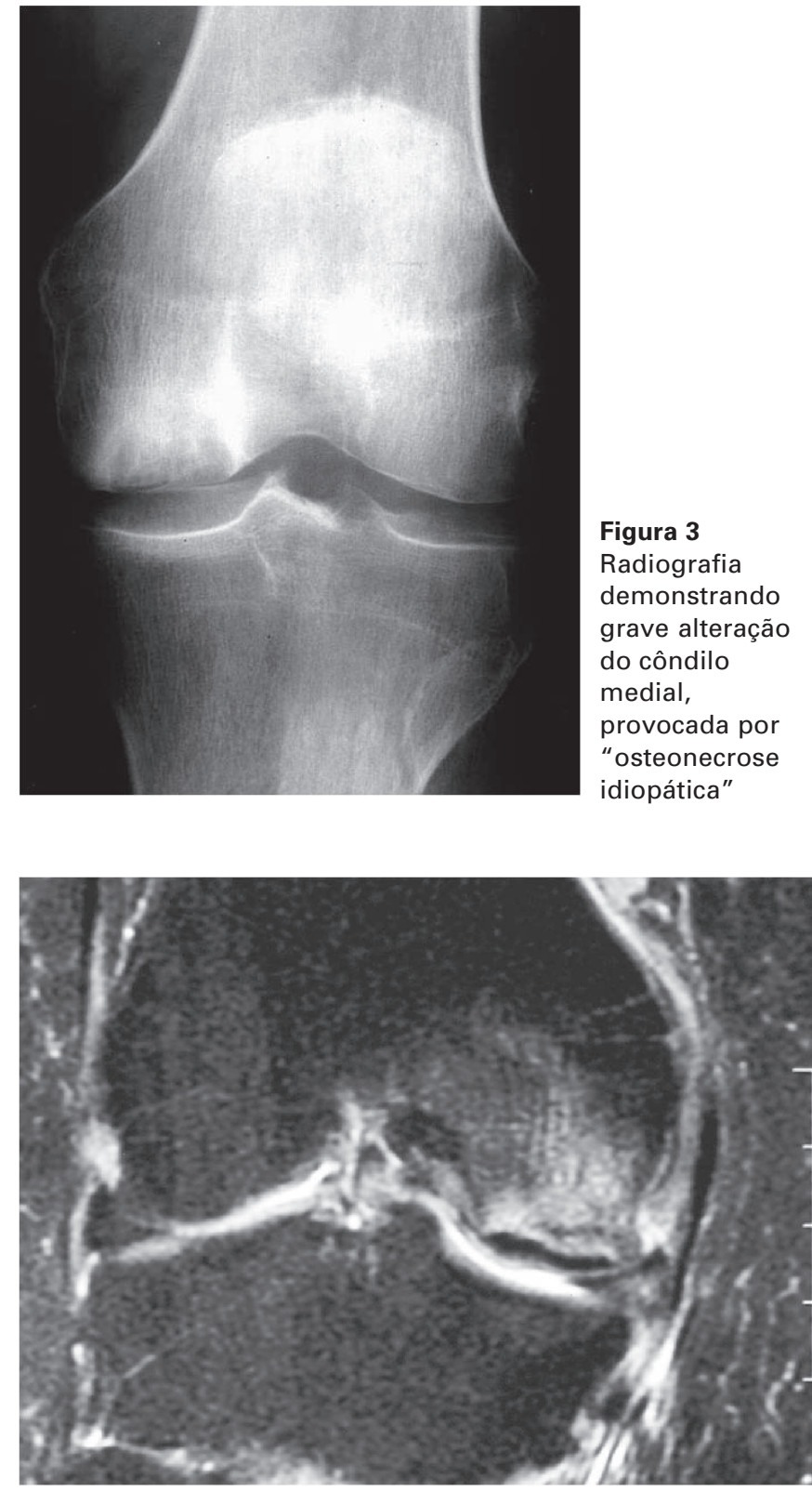

Figura 4 - RM demonstrando "osteonecrose idiopática" do côndilo femoral medial. Nota-se subluxação do menisco medial e traço de fratura.

A reabilitação deve ser prescrita no sentido de corrigir as retrações musculares que determinam a atitude em flexão e a melhora da potência muscular.

A orientação para atividade física freqüente e correção da osteoporose possibilitará melhor evolução destes pacientes. Esta conduta conservadora resolve a maioria dos casos. 

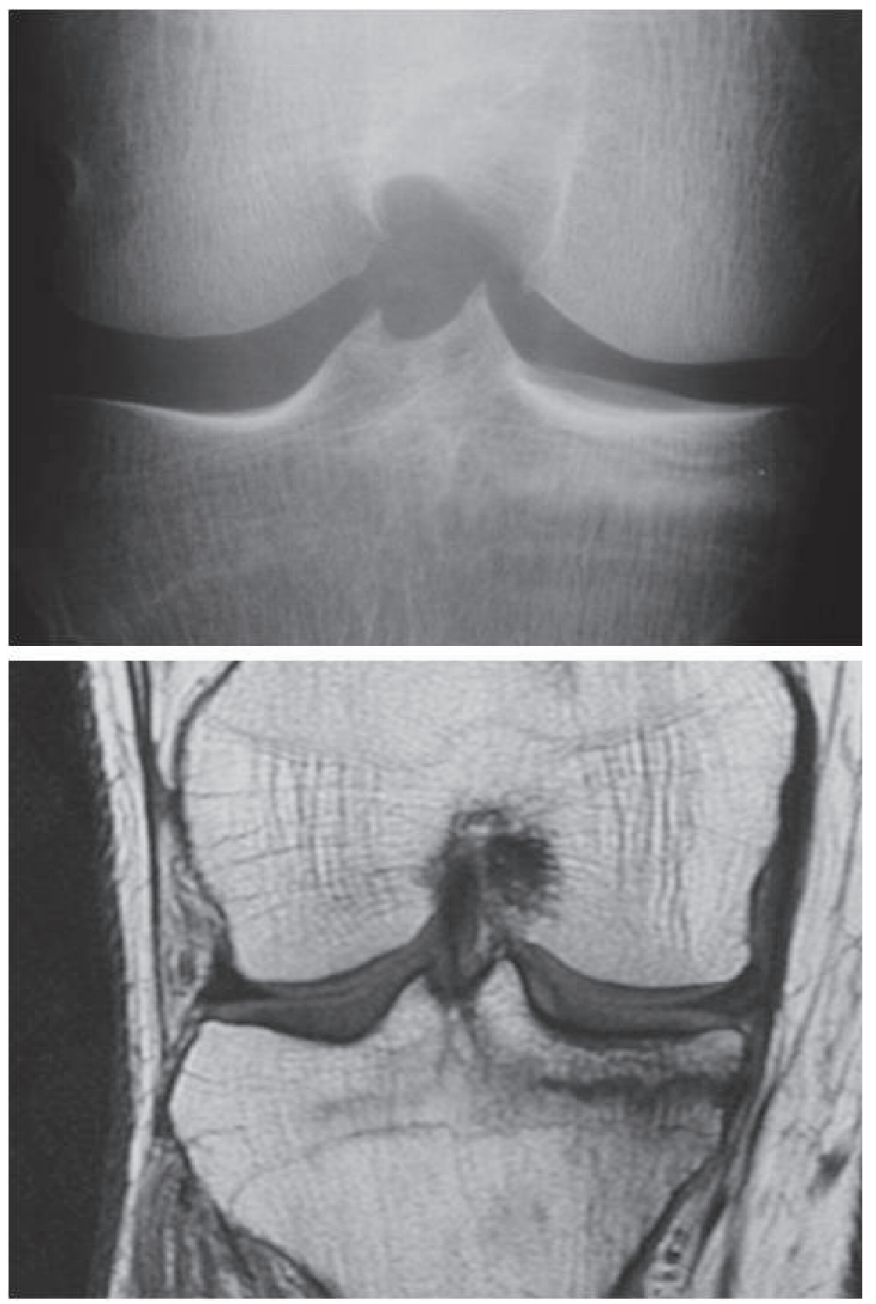

Figura 5 - Radiografia demonstrando traço de fratura por fadiga do planalto tibial medial, com correspondente RM, que demonstra de forma mais clara o traço de fratura por fadiga

A meniscectomia parcial trará melhora dos sintomas nos portadores de lesão meniscal, que apresentam recorrência dos sintomas pela ação mecânico-irritativo do fragmento meniscal. A sobrecarga do compartimento afetado piora o quadro evolutivo quando ocorre desvio de eixo, fato relativamente comum nesta faixa etária.

A osteotomia de alinhamento deve ser considerada, especialmente nos casos de osteonecrose idiopática em fase inicial.

Nos pacientes portadores de osteonecrose em fase avançada (III ou IV de Ahlbäck) está contra-indicado o tratamento conservador e sugere-se a substituição protética devido à grave destruição do compartimento afe- tado; nos casos mais avançados, sugere-se a artroplastia total.

\section{DISCUSSÃO}

A etiologia da dor aguda no joelho é basicamente decorrente de insuficiência das estruturas anatômicas; seu quadro clínico difere completamente daquele determinado pela osteoartrose.

A dor aguda é sempre unilateral e ocorre na maioria dos casos em pacientes do sexo feminino, após a quinta década de vida, e na região medial do joelho (local de maior carga). A osteoartrose, no entanto, não apresenta clara incidência em mulheres; em geral é bilateral, ocorre com desvio de eixo importante e os sintomas são de evolução crônica, com piora progressiva do quadro clínico.

O exame radiológico da artrose sintomática demonstra alterações articulares degenerativas, com pinçamento articular e desvio do eixo, enquanto que nos pacientes com dor aguda do joelho apresenta-se próximo do normal.

A lesão meniscal por fadiga é um dos tipos de lesão mais freqüente nos pacientes após a sexta década de vida.

A chamada osteonecrose idiopática do joelho é, na verdade, uma lesão por insuficiência ou fadiga. A imagem da RM é totalmente diferente da osteonecrose avascular que ocorre por uso de corticóide, alcoolismo, lúpus, hemoglobinopatias, pacientes portadores de HIV, como descrevem Lecouvet et al ${ }^{(8)}$. Gil et al consideram que a etiologia é traumática, decorrente de fratura por estresse agravada pela presença de lesão meniscal instável(4).

A fratura por fadiga, algumas vezes visível no exame radiográfico simples, é mais freqüente em atletas, mas não é rara em pacientes portadores de osteoporose, situação clínica comum em pacientes após a sexta década de vida ${ }^{(9)}$.

Embora com sintomas semelhantes, alguns pacientes não apresentam imagens características de uma das lesões descritas acima, ou então apresentam apenas osteoporose localizada. Lecouvet et al acreditam ser estas lesões semelhantes às por fadiga, porém de intensidades e resposta clínica diferentes ${ }^{(8)}$. 
Os pacientes portadores de dor aguda no joelho têm sintomatologia exuberante e na evolução desta queixa, ocorre manifestação da insuficiência de outras estruturas do joelho. São freqüentes os casos de osteonecrose idiopática do joelho (OIJ) com fratura por estresse $^{(6-7)}$. Yamamoto et al demonstraram a ocorrência de fraturas subcondrais em casos de dor aguda que haviam sido rotulados de $\mathrm{OIJ}^{(10)}$.

Não são raros os casos de lesão meniscal (LM) que após a correção cirúrgica evoluem para OIJ ${ }^{(11)}$. Possivelmente trata-se de uma mesma afecção, a "síndrome da insuficiência do joelho" (SIJ), que apresenta diversas manifestações clínicas e patológicas determinadas pelas características individuais. A semelhança do quadro clínico é muito sugestiva e a localização anatômica das lesões nos faz acreditar nesta suposição. As imagens de RM com edema ósseo presente, associado ou não a traço de fratura, sugere fortemente tratar-se de lesão provocada pela mesma condição patológica.

Lecouvet et al dividem as lesões com sinais de edema ósseo em dois grupos: o primeiro associado a condições que determinam osteoporose isoladamente e o segundo aos casos em que esta osteoporose se associa a traços de fratura ${ }^{(8)}$. Lequesne considera que são fases de uma mesma afecção ${ }^{(12)}$.

$\mathrm{O}$ tratamento visa adequar o joelho a insuficiência estrutural, procurando melhorar a postura e a musculatura destes pacientes. Nos casos mais sintomáticos, a proteção da articulação com uso de muletas é bastante útil, tendo importante efeito analgésico.

A meniscectomia em casos de sintomas decorrentes da presença de fragmentos traz alivio dos sintomas para a maioria dos pacientes ${ }^{(2)}$. Por tratar-se de lesão com- plexa e de certa forma evolutiva, pode ocorrer pósmeniscetomia, a evolução para a chamada osteonecrose idiopática ${ }^{(11)}$. A possibilidade desta ocorrência deve ser alertada ao paciente e poderá ser evitada com a proteção da articulação pós-meniscectomia, usando muletas e carga parcial sobre o membro afetado, além de programa de reabilitação para correção do flexo e da insuficiência do quadríceps.

Nos casos de osteonecrose idiopática, a osteotomia de alinhamento ou a substituição protética devem ser indicadas. A osteotomia traz melhora rápida dos sintomas e deve ser realizada precocemente quando não há sinais de destruição da superfície articular. Quando a RM demonstra destruição da superfície articular, a melhor indicação é a substituição protética.

Preferirmos a substituição com prótese unicompartimetal ${ }^{(13)}$, pois nestes casos a área afetada é, a princípio, de apenas um dos compartimentos. A evolução leva à destruição progressiva de toda a articulação. Nesses casos, a artroplastia total é a melhor opção.

\section{COMENTÁRIOS FINAIS}

Acreditamos, como Lequesne, que a lesão meniscal por fadiga, a osteonecrose idiopática e a fratura por fadiga sejam a mesma condição patológica que se apresenta com fases diferentes de evolução.

Melhores estudos anatomopatológicos e experimentais deverão ser desenvolvidos, para melhor compreensão. Sugerimos que sempre que um paciente se apresente com queixa de dor aguda no joelho, sem causa traumática, com idade acima de 50 anos, a "síndrome de insuficiência do joelho" é a opção que deve ser considerada no diagnóstico diferencial.

\section{CRÍTICA}

O artigo em questão esclarece pontos importantes sobre essa comum afecção dos joelhos. É importante lembrar o início súbito da afecção sem causa traumática ou um trauma de energia leve como um simples movimento com os joelhos em flexão exagerada. A ressonância magnética oferece diagnóstico mais preciso e nos propiciou melhor entendimento dessa patologia. A meniscectomia parcial necessita ser criteriosamente indicada e o paciente esclarecido dos resultados desse procedimento. $\mathrm{O}$ artigo oferece um correto conceito de que as diferentes patologias de dor aguda no joelho idoso pertencem a uma mesma entidade patológica em diferentes fases de evolução.

Prof. Dr. MoIsÉs COHEN

Prof. Adjunto e Chefe do Centro de Traumatologia do Esporte do Departamento de Ortopedia e Traumatologia da UNIFESP 


\section{REFERÊNCIAS}

1. Slemenda C, Brandt KD, Heilman DK, Mazzuca S, Braunstein EM, Katz BP, Wolinsky FD. Quadriceps weakness and osteoarthritis of the knee. Ann Intern Med. 1997;127(2):97104.

2. Camanho GL. Lesão meniscal no paciente idoso. Rev Hosp Clin Fac Med Univ Sao Paulo. 1997;52(3):127-31.

3. Ahlbäck S, Bauer GC, Bohne WH. Spontaneous osteonecrosis of the knee. Arthritis Rheum. 1968;11(6):705-33.

4. Gil HC, Levine SM, Zoga AC. MRI findings in the subchondral bone marrow: a discussion of conditions including transient osteoporosis, transient bone marrow edema syndrome, SONK, and shifting bone marrow edema of the knee. Semin Musculoskelet Radiol. 2006;10(3):177-86.

5. Pape D, Seil R, Fritsch E, Rupp S, Kohn D. Prevalence of spontaneous osteonecrosis of the medial femoral condyle in elderly patients. Knee Surg Sports Traumatol Arthrosc. 2002; 10(4):233-40.

6. Narváez JA, Narváez J, De Lama E, Sánchez A. Spontaneous osteonecrosis of the knee associated with tibial plateau and femoral condyle insufficiency stress fracture. Eur Radiol. 2003; 13(8):1843-8.
7. Kidwai AS, Hemphill SD, Griffiths HJ. Radiologic case study. Spontaneous osteonecrosis of the knee reclassified as insufficiency fracture. Orthopedics. 2005;28(3):236, 333-6.

8. Lecouvet FE, Malghem J, Maldague BE, Vande Berg BC. MR imaging of epiphyseal lesions of the knee: current concepts, challenges, and controversies. Radiol Clin North Am. 2005; 43(4):655-72, vii-viii.

9. Zanetti M, Romero J, Dambacher MA, Hodler J. Osteonecrosis diagnosed on MR images of the knee. Relationship to reduced bone mineral density determined by high resolution peripheral quantitative CT. Acta Radiol. 2003;44(5):525-31.

10. Yamamoto T, Bullough PG. Spontaneous osteonecrosis of the knee: the result of subchondral insufficiency fracture. J Bone Joint Surg Am. 2000;82(6):858-66.

11. Muscolo DL, Costa-Paz M, Ayerza M, Makino A. Medial meniscal tears and spontaneous osteonecrosis of the knee. Arthroscopy. 2006;22(4):457-60.

12. Lequesne M. Transient osteoporosis of the hip. A nontraumatic variety of Südeck's atrophy. Ann Rheum Dis. 1968;27(5):46371.

13. Camanho GL, Rossetti AC, Camanho LF, Albuquerque RP. Artroplastia unicompartimental do joelho no tratamento da osteonecrose primária do côndilo femoral medial. Rev Bras Ortop. 2004;39(9):486-91.

\footnotetext{
Declaração de inexistência de conflitos de interesse: Declaro que o presente estudo não possui conflito de interesse, nem houve suporte financeiro.
} 